EPSC Abstracts,

Vol. 4, EPSC2009-579, 2009

European Planetary Science Congress,

(C) Author(s) 2009

\title{
Water and related chemistry in the Solar System. A Guaranteed Time Key Programme for Herschel
}

P. Hartogh (1), J. Crovisier (2), E. Lellouch (2), M. Banaszkiewicz (3), F. Bensch (4,5), E.A. Bergin (6), F. Billebaud (7,8), N. Biver (2), G.A. Blake (9), M.I. Blecka (3), J. Blommaert (10), D. Bockelée-Morvan (2) , T. Cavalié (1), J. Cernicharo (11), R. Courtin (2), G. Davis (12), L. Decin (10), P. Encrenaz (13), T. Encrenaz (2), T. de Graauw (14/15/16), A. Gonzáles (1,17), D. Hutsemékers (18), C. Jarchow (1), E. Jehin (18), M. Kidger (19), M. Küppers (20), A. de Lange (14), L.-M. Lara (17), D.C. Lis (9), R. Lorente (19) J. Manfroid (18), A.S. Medvedev (1), R. Moreno (2), D.A. Naylor (21), G. Orton (22), G. Portyankina (23), M. Rengel (1), H. Sagawa (1), M. SánchezPortal (19), R. Schieder (24), S. Sidher (25), D. Stam (14), B. Swinyard (25), S. Szutowicz (3), N., Thomas (23), G. Thornhill (19), B. Vandenbussche (10), E. Verdugo (19) and H. Walker (25)

(1) Max Planck Institute for Solar System Research, Katlenburg-Lindau, Germany (Hartogh@mps.mpg.e) (2) LESIA, Observatoire de Paris, France, (3) Space Research Centre, Polish Academy of Sciences, Warsaw, Poland, (4) Argelander Institute for Astronomy, University of Bonn, Germany, (5) DLR, German Aerospace Centre, BonnOberkassel, Germany, (6) Astronomy Department, University of Michigan, USA, (7) Université de Bordeaux, Laboratoire d'Astrophysique de Bordeaux, France (8) CNRS/INSU, UMR 5804, Floirac, France, (9) California Institute of Technology, Pasadena, USA, (10) Instituut for Sterrenkunde, Katholieke Universiteit Leuven, Belgium, (11) Laboratory of Molecular Astrophysics, CAB-CSIC. INTA, Madrid, Spain, (12) Joint Astronomy Center, Hilo, USA, (13) LERMA, Observatoire de Paris, France (14) SRON, Netherlands Institute for Space Research, Netherlands, (15) Leiden University, Netherlands, (16) Joint ALMA Observatory, Chile, (17) Instituto de Astrofisica de Andalucia, Granada, Spain, (18) Institute d'Astrophysique et de Geophysique, Université de Liège, Belgium, (19) Herschel Science Operations Centre, European Space Astronomy Centre, European Space Agency, Madrid, Spain, (20) Rosetta Science Operations Centre, European Space Astronomy Centre, European Space Agency, Madrid, Spain, (21) Department of Physics and Astronomy, University of Lethbridge, Canada, (22) Jet Propulsion Laboratory, California Institute of Technology, Pasadena, USA, (23) Physikalisches Institut, University of Bern, Switzerland, (24) 1st Physics Institute, University of Cologne, Germany, (25) Space Science \& Technology Department, Rutherford Appleton Laboratory, UK.

\begin{abstract}
This Guaranteed Time Key Programme, approved by the European Space Agency, aims at determining the distribution, the evolution and the origin of water in Mars, the Outer Planets, Titan, Enceladus and Comets, using the three Herschel instruments HIFI, PACS and SPIRE. It addresses the broad topic of water and its isotopologues in planetary and cometary atmospheres. The nature of cometary activity and the thermodynamics of cometary comae will be investigated by studying water excitation in a sample of comets. The $\mathrm{D} / \mathrm{H}$ ratio, the key parameter for constraining the origin and evolution of Solar System species, will be measured for the first time in a Jupiter-family comet. A comparison with existing and new measurements of $\mathrm{D} / \mathrm{H}$ in Oort-cloud comets will constrain the composition of pre-solar cometary grains and possibly the dynamics of the protosolar nebula. New measurements of $\mathrm{D} / \mathrm{H}$ in Giant
\end{abstract}

Planets, similarly constraining the composition of proto-planetary ices, will be obtained.

The $\mathrm{D} / \mathrm{H}$ and other isotopic ratios, diagnostic of Mars' atmosphere evolution, will be accurately measured in $\mathrm{H}_{2} \mathrm{O}$ and $\mathrm{CO}$. The role of water vapour in Mars' atmospheric chemistry will be studied by monitoring vertical profiles of $\mathrm{H}_{2} \mathrm{O}$ and $\mathrm{HDO}$ and by searching for several other species (and $\mathrm{CO}$ and $\mathrm{H}_{2} \mathrm{O}$ isotopes). A detailed study of the source of water in the upper atmosphere of the Giant Planets and Titan will be performed. By monitoring the water abundance, vertical profile, and input fluxes in the various objects, and when possible with the help of mapping observations, we will discriminate between the possible sources of water in the Outer Planets (interplanetary dust particles, cometary impacts, and local sources). In addition to these inter-connected objectives, serendipitous searches may further enhance our knowledge of the composition of planetary and cometary atmospheres. For further details see: http://www.mps.mpg.de/projects/herschel/HssO/ 